\title{
CCl-779 (Temsirolimus) exhibits increased anti-tumor activity in low EGFR expressing HNSCC cell lines and is effective in cells with acquired resistance to cisplatin or cetuximab
}

\author{
Franziska Niehr ${ }^{1,2}$, Wilko Weichert ${ }^{3,4}$, Albrecht Stenzinger ${ }^{3}$, Volker Budach ${ }^{1,2}$ and Ingeborg Tinhofer ${ }^{1,2,5^{*}}$
}

\begin{abstract}
Background: The mammalian target of rapamycin (mTOR) signaling pathway plays a pivotal role in numerous cellular processes involving growth, proliferation and survival. The purpose of this study was to investigate the anti-tumoral effect of the mTOR inhibitor (mTORi) CCI-779 in HNSCC cell lines and its potency in cisplatin- and cetuximab-resistant cells.

Methods: A panel of 10 HNSCC cell lines with differences in TP53 mutational status and basal cisplatin sensitivity and two isogenic models of acquired resistance to cisplatin and cetuximab, respectively were studied. Cell survival after treatment with CCl-779, cisplatin and cetuximab alone or in combination was determined by MTT assays. Potential predictive biomarkers for tumor cell sensitivity to CCl-779 were evaluated.

Results: We observed considerable heterogeneity in sensitivity of HNSCC cell lines to CCI-779 monotherapy. Sensitivity was observed in TP53 mutated as well as wild-type cell lines. Total and p-EGFR expression levels but not the basal activity of the mTOR and MAPK signaling pathways were correlated with sensitivity to CCl-779. Resistant cells with increased EGFR activation could be sensitized by the combination of CCl-779 with cetuximab. Interestingly, cell lines with acquired resistance to cisplatin displayed a higher sensitivity to $\mathrm{CCl}-779$ whereas cetuximab-resistant cells were less sensitive to the drug, but could be sensitized to CCl-779 by EGFR blockade.

Conclusions: Activity of CCI-779 in HNSCC cells harboring TP53 mutations and displaying a phenotype of cisplatin resistance suggests its clinical potential even in patients with dismal outcome after current standard treatment. Cetuximab/mTORi combinations might be useful for treatment of tumors with high expression of EGFR/p-EGFR and/or acquired cetuximab resistance. This combinatorial treatment modality needs further evaluation in future translational and clinical studies.
\end{abstract}

Keywords: HNSCC, mTOR, EGFR, Temsirolimus, CCI-779, Cetuximab, Cisplatin, Resistance

\section{Background}

Cancer of the head and neck region is the sixth most common cause for cancer-related mortality worldwide with 600,000 new cases and 300,000 deaths per year. Tobacco and heavy alcohol drinking are the most important risk factors [1,2]. Depending on the disease stages,

\footnotetext{
*Correspondence: ingeborg.tinhofer@charite.de

${ }^{1}$ Translational Radiooncology Laboratory, Department of Radiooncology and Radiotherapy, Charité University Hospital, Berlin, Germany

${ }^{2}$ German Cancer Consortium (DKTK), German Cancer Research Center (DKFZ) Heidelberg, Partner Site Berlin, Berlin, Germany

Full list of author information is available at the end of the article
}

surgery, radiotherapy, and chemotherapy, alone or in combinations, have been used as therapeutic options over the past decades. New treatment strategies such as epidermal growth factor receptor (EGFR) inhibition by cetuximab have been shown to prolong survival, but 5-year survival rates of patients with locally advanced cancers are still at $50 \%$ or below [3]. Therefore, new therapeutic approaches like second generation- or multi-target tyrosine kinase inhibitors, proteasome inhibitors, hypoxia-modifying agents, or antiangiogenic agents have been or are being evaluated in clinical trials [4-7]. Among these, one of the most 
promising approaches is the inhibition of the phosphatidylinositol 3-kinase-related kinase (PI3K) pathway, since it represents the most frequently mutated oncogenic pathway in HNSCC [8].

The mammalian target of rapamycin (mTOR) is a serine/threonine protein kinase and belongs to the PI3Krelated kinase protein family. It is involved in many cellular processes like cell growth, proliferation, and survival [9]. Different mTORi including rapamycin, RAD-001 (everolimus) and CCI-779 (cell cycle inhibitor-779, temsirolimus), but also dual mTOR inhibitors that target both of the two mTOR complexes, mTORC1 and mTORC2 have been evaluated [10,11]. In HNSCC, rapamycin [12,13], everolimus [14] and temsirolimus [15], as well as dual inhibitors [16] have already shown promising effects in vitro and in vivo. Their combination with irradiation, cisplatin or reagents targeting the epidermal growth factor receptor (EGFR) like cetuximab and erlotinib $[17,18]$ has been evaluated in preclinical models $[11,19,20]$ and clinical trials [21]. However, the molecular mechanisms of sensitivity/resistance of HNSCC cells to mTORi remain poorly characterized and no predictive biomarker for patient selection has been established so far.
To evaluate the activity of the mTORi CCI-779 in HNSCC in more detail and to identify predictive biomarkers, the genetic profile, as well as mRNA- and protein expression of genes involved in the mTOR pathway were characterized in $10 \mathrm{HNSCC}$ cell lines and correlated to their sensitivity to CCI-779. Furthermore, the effectiveness of CCI-779 in cells with primary/acquired resistance to cisplatin and cetuximab was evaluated.

\section{Results and discussion}

Characterization of the growth-inhibitory potential in HNSCC cell lines

In all tested HNSCC cell lines treatment with CCI-779 $(100 \mathrm{ng} / \mathrm{ml})$ for $72 \mathrm{~h}$ reduced cell viability, ranging from a reduction of $69 \%$ in UM-SCC-11B cells down to $40 \%$ in UT-SCC-15 cells compared to solvent-treated cells (Figure $1 \mathrm{~A}$ ). In long-term studies (7 days of treatment) we were able to clearly distinguish sensitive from resistant cells, with UT-SCC-23 representing the most resistant $(95 \%$ viability after treatment with $100 \mathrm{ng} / \mathrm{ml})$ and UM-SCC-25 (16\% viability) the most sensitive cell line (Figure $1 \mathrm{~B}$ ). Of note, the IC50 of cisplatin, a widely used radiosensitizer in HNSCC, only slightly differed between

(A)

(B)
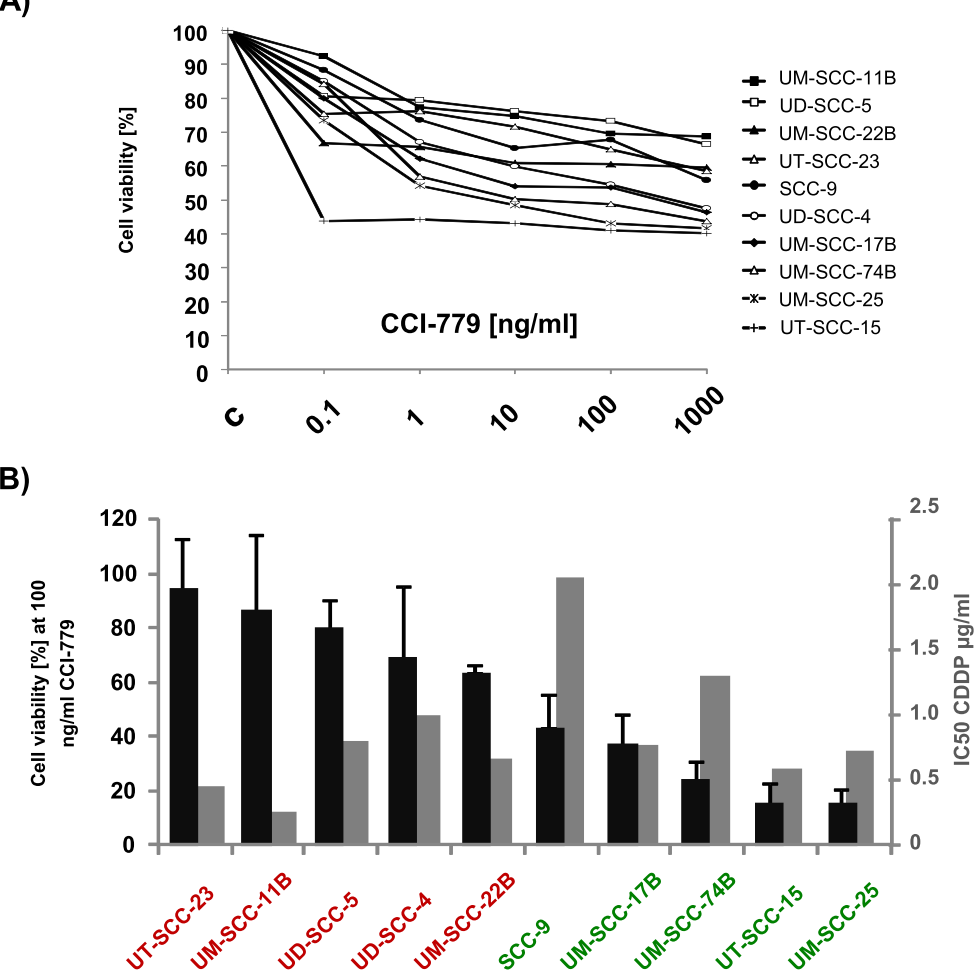

Figure 1 CCI-779 shows an inhibitory effect on cell survival of cisplatin-sensitive and -resistant HNSCC cell lines. (A) HNSCC cell lines were treated with increasing doses of CCl-779 (0.1 to $1000 \mathrm{ng} / \mathrm{ml})$. After $72 \mathrm{~h}$ cell survival was determined by the MTT assay. (B) The same cell lines were treated with CCl-779 at $100 \mathrm{ng} / \mathrm{ml}$ for 7 days in long-term MTT assays (black bars, left scale). Their IC50 for cisplatin was as well evaluated in MTT assays (grey bars, right scale). Cells were then grouped in CCl-779-resistant (red) and -sensitive (green) lines using a cutoff of 50\% cell survival. (A, B) Survival fractions for given treatments were calculated on the basis of the survival of non-treated cells. Each sample was done in triplicate and experiments were performed thrice. 
these two cell lines ( 0.5 and $0.7 \mu \mathrm{g} / \mathrm{ml}$, respectively), speaking against cross-resistance between the two drugs (Figure $1 \mathrm{~B}$ ). This finding together with the high efficiency in most of the cell lines tested speaks for CCI-779 as potentially effective therapeutic option in HNSCC cells with primary resistance to cisplatin.

Neither TP53 status nor mutations in HNSCC-associated oncogenic pathways predict sensitivity to CCI-779.

In search for predictive biomarkers for mTOR inhibition the involvement of TP53 and other genes from HNSCC-related oncogenic pathways for CCI-779 sensitivity was determined. For this purpose, TP53 gene and transcript sequences were analyzed by panel nextgeneration sequencing (NGS) and Sanger sequencing, respectively. In addition, the expression and functional status of the p53 protein was determined. Sequencing revealed distinct mutations of TP53 in the cell lines tested, with Sanger sequencing and panel NGS giving the same results (Tables 1 and 2). The cyclin-dependent kinase inhibitor 1 (p21) represents one of the p53 targets. Its elevated expression after irradiation served as a readout for functional activity of p53. There was no significant correlation observed between the expression of p53 transcripts $(\mathrm{p}=.988)$ or proteins $(\mathrm{p}=.990)$ or its transcriptional function $(\mathrm{p}=.607)$ and the sensitivity of cells to CCI-779 (Table 1). Previously, reduced sensitivity of HNSCC cell lines carrying a TP53 mutation to a dual PI3K/mTOR inhibitor was reported [22]. In line with this previous study, wt TP53 was exclusively detected in the group of sensitive cell lines, displaying decreased viability after treatment with $100 \mathrm{ng} / \mathrm{ml}$ of temsirolimus compared to mutated cells (mean viability $\pm \mathrm{SD}$ : wt TP53 group [ $\mathrm{N}=3], 0.36 \pm 0.19$ vs mutated TP53 group $[\mathrm{N}=7], 0.65 \pm 0.27)$. However, this difference in viability did not reach significance level $(\mathrm{p}=.139)$ which might be due to the limited number of cell lines carrying wt TP53 in our subset.
Panel NGS revealed further mutations in key oncogenic pathways including receptor tyrosine kinase, PI3K or MAPK signaling in our cell lines (Table 2). Mutations were also found in genes involved in cell cycle control and cell death regulation, as well as in the tumor suppressor SMAD4 and the transmembrane receptor gene NOTCH1. None of these mutations was associated with sensitivity to CCI-779. Since only one cell line within our panel carried a PI3KCA mutation, the involvement of this alteration in sensitivity to mTORi, as discussed in other studies $[8,22]$, could not be addressed.

CCI-779 sensitivity does not correlate with mTOR/MAPK signaling pathway activity.

As potential biomarker for mTORi activity, the basal activation of the mTOR or MAPK-pathway has been discussed as well as the ability of mTORi to inhibit downstream signaling of mTOR. It is thought that high activity of the mTOR pathway renders tumor cells more dependent on mTOR and results in higher sensitivity to mTORi, whereas high activity of MAPK signaling has been associated with reduced sensitivity to mTOR inhibition $[8,23,24]$. We therefore evaluated basal protein expression levels of p-S6 (a downstream target of mTOR) and p-Erk (a member of the MAPK pathway) as well as their expression levels after mTOR inhibition in four cell lines with highest and lowest sensitivity to CCI-779 (Figure 2 A). No correlation could be found between sensitivity of cells to CCI-779 and their basal expression levels of $\mathrm{p}$-S6 or $\mathrm{p}$-Erk, with all cell lines expressing phosphorylated, activated forms of these kinases. Furthermore, CCI-779 was able to inhibit phosphorylation of S6, downstream of p70S6K in all tested cell lines regardless of their sensitivity to the drug in the proliferation assays (Figure $2 \mathrm{~A}$ ). To validate these findings, we included all cell lines used in the proliferation assays. Again, no correlation was found between basal expression of total or activated proteins

Table 1 Characteristics of HNSCC cell lines

\begin{tabular}{|c|c|c|c|c|c|}
\hline Cell line & TP53 genotype & p53 transcript & p53 protein & function & $\mathrm{CCl}-779100 \mathrm{ng} / \mathrm{ml}$ \\
\hline UT-SCC-23 & Loss of transcript & - & - & - & 95 \\
\hline UM-SCC-11B & missense mutation & + & + & - & 87 \\
\hline UD-SCC-5 & missense mutation & + & + & - & 81 \\
\hline UD-SCC-4 & delete, fs (stop codon) & + & - & - & 70 \\
\hline UM-SCC-22B & missense mutation & + & + & - & 64 \\
\hline SCC-9 & delete, fs & + & + (trunc.) & - & 44 \\
\hline UM-SCC-17B & wt & + & + & + & 38 \\
\hline UM-SCC-74B & wt & + & + & + & 25 \\
\hline UT-SCC-15 & Incorrectly spliced transcript, fs & + & + & - & 16 \\
\hline UM-SCC-25 & wt & $(+)$ & - & - & 16 \\
\hline
\end{tabular}

Cell lines were ordered by their resistance to $\mathrm{CCl}-779$ at $100 \mathrm{ng} / \mathrm{ml}$. Presented are their TP53 genotype, transcript- and protein expression. P53 function was assessed by measuring p21 and porphobilinogen deaminase (PBGD) mRNA expression before and after irradiation, using a cut-off of 1.5 fold induction. 
Table 2 Mutations identified by panel next-generation sequencing for cell lines (upper panel) and resistance models (lower panel) used in this study

\begin{tabular}{|c|c|c|c|c|c|c|c|}
\hline Cell line & Cell cycle control & TP53 & Cell death regulation & RTK signaling & PI3K-akt signaling & MAPK-signaling & Others \\
\hline UT-SCC-23 & & DEL & & & & & SMAD4,FAT1 \\
\hline UM-SCC-11B & & Cys110Ser & & & & & \\
\hline UD-SCC-5 & & His47Tyr & & ERBB4, PDGFR & & & FAT1, PCDH15 \\
\hline UD-SCC-4 & & DEL & & $K D R, M Y C$ & & & FAT1, PCDH15 \\
\hline UM-SCC-22B & & Tyr88Cys & CASP8 & KIT & & & NOTCH1, SMAD4, FAT1, PCDH15, \\
\hline SCC-9 & & Val142fs & & $K D R, R E T$ & & & FAT1, Notch1, PCDH15 \\
\hline UM-SCC-17B & & & & $E G F R, K D R, K I T$ & PIK3CA & HRAS & SMAD4, FAT1 \\
\hline UM-SCC-74B & & & & $K D R, K R A S$ & & & CDH1, FAT1, NOTCH1, PCDH15 \\
\hline UT-SCC-15 & CDKN2A & Arg282Trp & CASP8 & & & & CDH1, FAT1 \\
\hline UM-SCC-25 & CDKN2A & & & ERBB4 & & & SMAD4, FAT1 \\
\hline $\mathrm{FaDu}_{\mathrm{CDDP}-\mathrm{S} / \mathrm{R}}$ & CDKN2A & Arg248Leu $2 \% \rightarrow 47 \%$ & & & & & SMAD4 \\
\hline UD-SCC-4 $4_{C D D P-S / R}$ & & DEL & & MYC, KDR $70 \% \rightarrow 100 \%$ & & & FAT1, PCDH15, NSD1 0\% $\rightarrow 38 \%$ \\
\hline UT-SCC-9 $9_{C E T-S / R}$ & CDKN2A & DEL & & MET $35 \% \rightarrow 50 \%$ & & & FAT1 \\
\hline UM-SCC-22B CET-S/R $_{\text {. }}$ & & Tyr88Cys & CASP8 & KIT & & & NOTCH1, SMAD4, FAT1, PCDH15 \\
\hline
\end{tabular}

Mutations which affect protein function, as predicted by the SIFT [41] and PolyPhen [42] program are presented in bold, percentages of allele frequency are given if they differed between parental and resistant cells. 
(A)
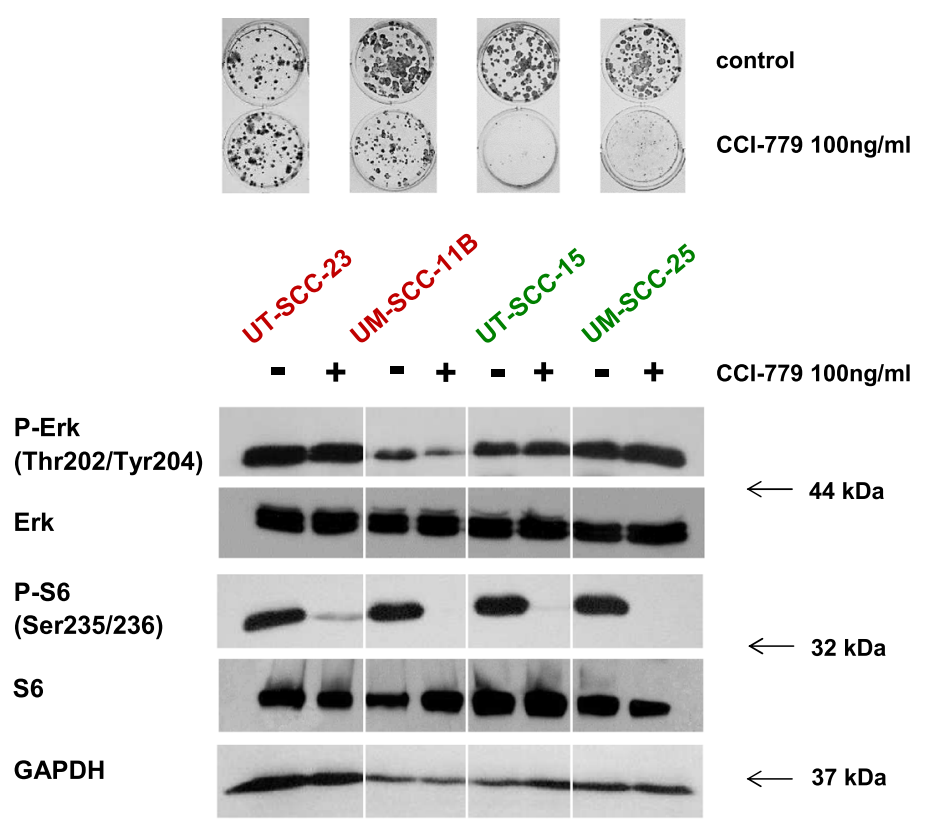

(B)

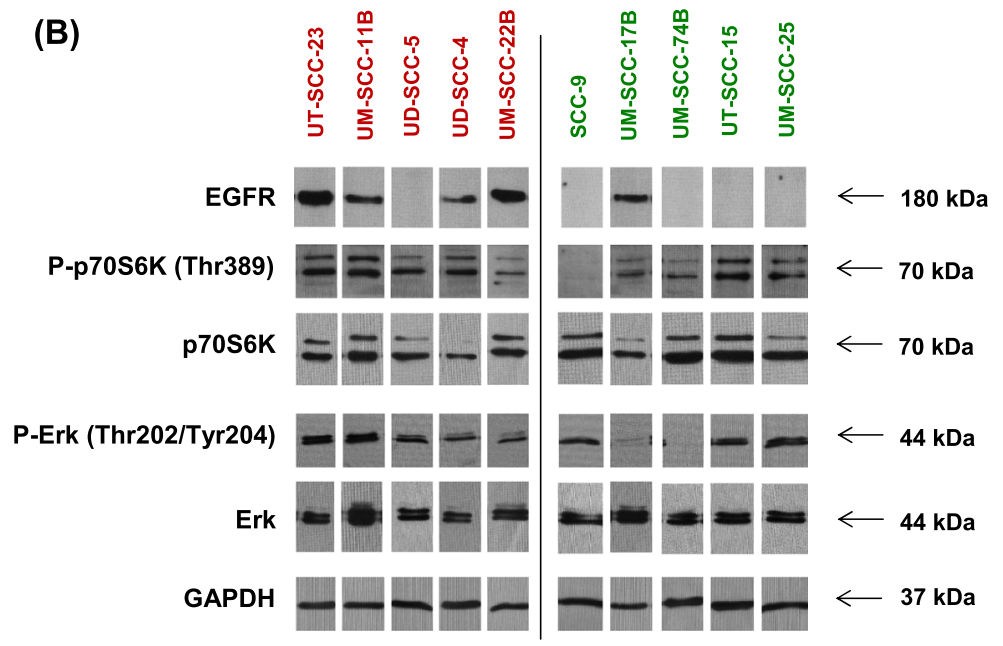

Figure 2 Resistance to $\mathrm{CCl}-779$ is associated with high EGFR expression but does not correlate with reduced activity of CCI-779 in blocking mTOR-signaling. (A) Two resistant (red, UT-SCC-23 and UM-SCC-11B) and two sensitive (green, UT-SCC-15 and UM-SCC-25) cell lines were treated with $100 \mathrm{ng} / \mathrm{ml} \mathrm{CCl-779}$ for $24 \mathrm{~h}$. The activation of the MAPK- (p-Erk) and mTOR- (p-S6) signaling pathways, as well as the expression of the housekeeper protein GAPDH were evaluated using Western Blot. (B) All 10 cell lines were analyzed regarding their basal expression levels of EGFR, p-Erk/Erk (MAPK pathway), and p-S6K/S6K (mTOR pathway) by Western Blot.

of Erk/p-Erk or p70S6K/p-p70S6K and sensitivity to CCI-779 (Figure 2 B).

HNSCC cells with primary resistance to CCI-779 display high EGFR expression and can be sensitized by EGFR blockade.

High EGFR protein and mRNA expression were detected almost exclusively in resistant cells (Figures $2 \mathrm{~B}$ and $3 \mathrm{~A})$. Interestingly, p-EGFR expression was elevated in the group of cell lines with reduced sensitivity to CCI-779 (Figure 3B; mean p-EGFR expression \pm SD: resistant group $[\mathrm{N}=5], 4.18 \pm 1.97$ vs sensitive group [ $\mathrm{N}=5], 1.78 \pm 0.29$ ).
Statistical analysis revealed borderline significance $(\mathrm{p}=$ 0.052 ) for this association. Basal MAPK- (p-Erk) and mTOR- (p-p70S6K) pathway activity did not differ between resistant and sensitive cells, therefore other downstream targets of EGFR might be involved in resistance to CCI-779. To test the hypothesis that overexpression of p-EGFR contributes to resistance to CCI-779, three resistant cell lines, expressing high levels of EGFR and p-EGFR, and three sensitive lines, expressing low EGFR/p-EGFR levels, were treated with increasing concentrations of CCI-779 alone or in combination with a 


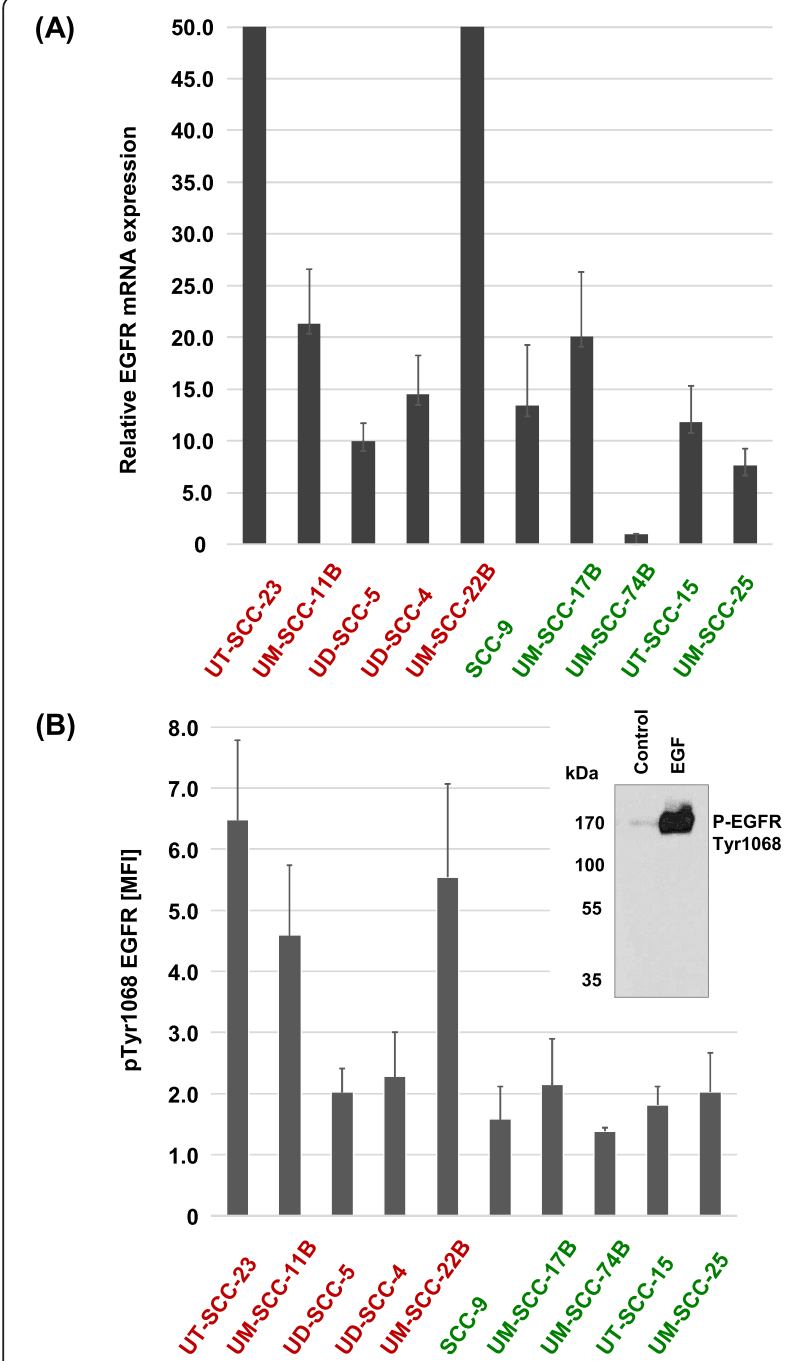

Figure 3 Cell lines resistant to $\mathrm{CCl}-779$ demonstrate higher expression levels of EGFR mRNA and elevated p-EGFR protein levels. (A) EGFR mRNA levels were measured by Real Time RT-PCR and correlated to the housekeeper gene Tubulin alpha-6. The relative EGFR mRNA expression was calculated using UM-SCC-74B with the lowest EGFR expression levels as reference and is given as fold levels. (B) To quantify the basal expression of phosphorylated, activated EGFR (p-EGFR Tyr 1068) flow cytometry was used. Results are given as the median specific fluorescence intensity (MFI) of cells stained with the specific antibody divided by the intensity of cells stained with the isotype control antibody.

fixed low dose of cetuximab. All of the resistant cell lines could be sensitized to CCI-779 by the combination whereas no further sensitization of the sensitive cell lines was observed (Figure 4). The greatest effect was seen in the most resistant cell line UT-SCC-23 with the highest EGFR/p-EGFR expression. Cell viability after CCI-779 treatment was reduced from $91 \%$ to $15 \%$ after combining CCI-779 with cetuximab. This finding suggests that patients with high EGFR/p-EGFR expressing tumors could benefit from a combined mTORi/anti-EGFR treatment. In support of these results, targeting of mTOR and EGFR in preclinical models of HNSCC has previously been shown to result in a higher growth inhibition than treatment with each agent alone $[18,25,26]$. Several studies have already been initiated for the identification of biomarkers of mTORi efficacy, with a focus on activation of the mTOR and the MAPK pathway before or after treatment (NCT00195299, CCI-779; NCT01195922, rapamycin; both advanced disease). Moreover, EGFR expression among others is being evaluated as biomarker for RAD001 activity in refractory recurrent locally advanced HNSCC (NCT01051791).

In our model of acquired cetuximab resistance, we showed that cetuximab-resistant cell lines either did not change their sensitivity or became more resistant to CCI-779 (temsirolimus) compared to their sensitive counterparts. This is in line with results from the PII MAESTRO study in which metastatic HNC patients with documented progression on cetuximab were treated with either temsirolimus alone or its combination with cetuximab [27]. This study revealed modest and short activity of temsirolimus as single agent in this scenario. The addition of temsirolimus to cetuximab was able to overcome acquired cetuximab resistance in only a small subgroup of patients (12.5\%), however, these responses were more durable than the response to the previous cetuximab-containing regimens [27]. In our model of acquired cetuximab resistance, the combined treatment of cetuximab-resistant cells with cetuximab and CCI-779 was more effective than CCI-779 alone, supporting potential clinical value of this combination. It will be important to evaluate in future studies whether expression levels of EGFR/p-EGFR which we established here as predictive factor for low CCI-779 sensitivity in cetuximab-naïve HNSCC cells might also be useful as predictive biomarker for clinical benefit of cetuximab/ temsirolimus combinations in the cetuximab-refractory setting.

HNSCC cells with acquired resistance to cisplatin or cetuximab show differential sensitivity to CCI-779 and can be resensitized by combinatorial treatment.

Cisplatin and cetuximab, each of them in combination with radiotherapy, are effective drugs in the primary treatment of locally advanced HNSCC. In the recurrent disease setting the tumor response to these agents is reduced, most likely due to selection of drug-resistant cells during primary treatment. To evaluate the potential of CCI-779 in the recurrent disease, we established four models of acquired resistance to cisplatin $(\mathrm{FaDu}$ and UD-SCC-4) and cetuximab (UT-SCC-9 and UM-SCC22B) by long-term treatment of HNSCC cell lines with increasing concentrations of the drugs (Figure $5 \mathrm{~A}$ and B). Interestingly, we observed that both cell lines with acquired cisplatin resistance $\left(\mathrm{FaDu}_{\mathrm{CDDP}-\mathrm{R}}\right.$ and UD-SCC- 

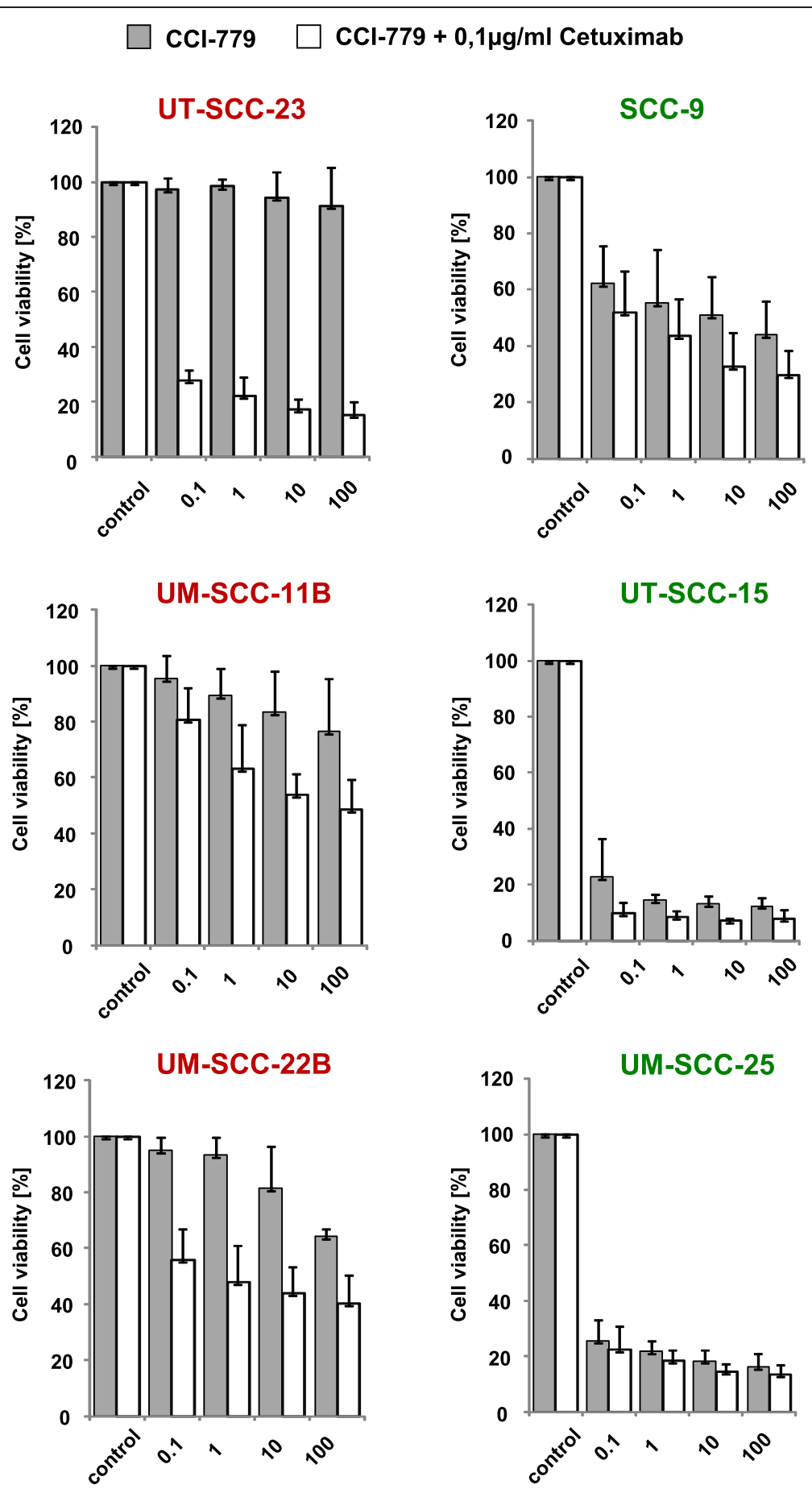

Figure 4 The combination of $\mathrm{CCl}-779$ and cetuximab augments the sensitivity of cells to treatment with the mTOR inhibitor alone. All 10 cell lines were treated with increasing doses of CCl-779 $(0.1-100 \mathrm{ng} / \mathrm{ml})$ and long-term cell viability (7 days) was measured using the MTT assay. Additionally, CCl-779 treatment was combined with cetuximab at a low dose $(0.1 \mu \mathrm{g} / \mathrm{ml})$, at which the drug itself showed only little effect. In three EGFR-expressing, CCl-779 resistant cell lines the combination led to decreased cell viability (left graphs). Cell viability of sensitive cells could not further be decreased by the combination (three representative cell lines, right graphs). Survival fractions after drug treatment were calculated on the basis of the survival of non-treated cells. 

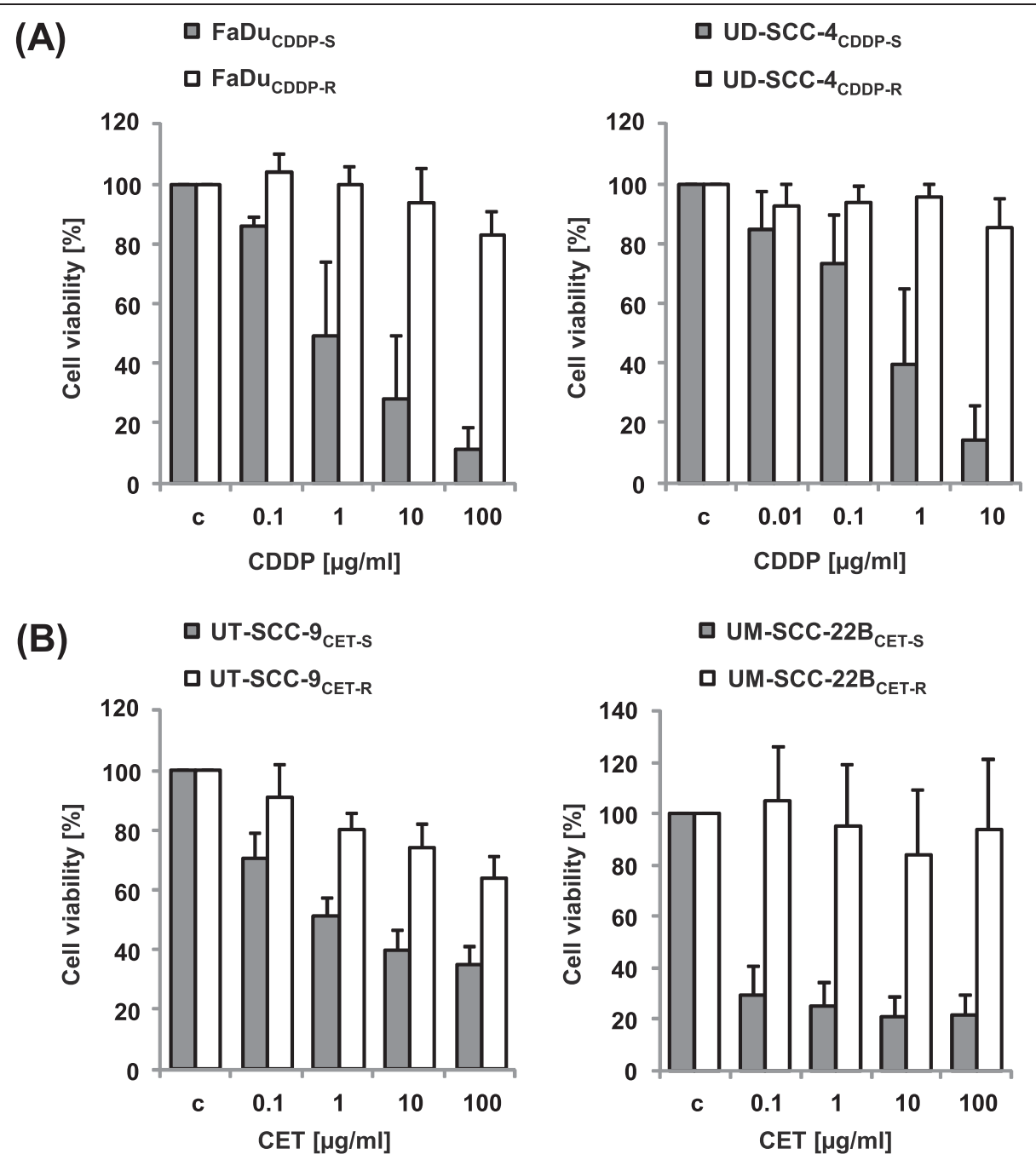

Figure 5 Long-term treatment of HNSCC cells with cisplatin or cetuximab selects resistant subclones. Cell lines were treated for 6-9 months with increasing doses of (A) cisplatin (FaDuCDDP-S, UD-SCC-4CDDP-S) or (B) cetuximab (UT-SCC-9CET-S, UM-SCC-22BCET-S). Doses were elevated if the cells showed a stable growth. The resulting resistant cells (CDDP-/CET-R) as well as their sensitive parental counterparts (CDDP-/CET-S) were used forfurther experiments.

$\left.4_{\text {CDDP-R }}\right)$ showed a better response to CCI-779 compared to their parental counterparts $\left(\mathrm{FaDu}_{\mathrm{CDDP}-\mathrm{S}}\right.$ and UD-SCC$\left.4_{\text {CDDP-S }}\right)$. In contrast, cetuximab-resistant cell lines either did not change their sensitivity (UM-SCC-22B $\mathrm{B}_{\mathrm{CET}-\mathrm{R}}$ ) or became more resistant to CCI-779 (UT-SCC-9 ${ }_{\text {CET-R }}$ ) compared to the parental cetuximab-sensitive cells (Figure 6).

By panel NGS analysis we detected an increased allele frequency of a specific TP53 exon mutation (Arg248Leu) in $\mathrm{FaDu}_{\mathrm{CDDP}-\mathrm{R}}$ that was already present in the parental cell line $\mathrm{FaDu}_{\mathrm{CDDP}-\mathrm{S}}$, indicating the selection of a preexisting subclone (Table 2). In the UD-SCC-4 ${ }_{\mathrm{CDDP}-\mathrm{R}}$ cell line, the selection of subclones harboring KDR (VEGFR2) and NSD1 mutations was observed. TP53 and $K D R$ mutations have been associated with cisplatin resistance $[28,29]$ and NSD1 is known to regulate NF$\kappa B$ [30] which has also been involved in resistance to cisplatin [31]. In one of the two cetuximab-resistant cell lines (UT-SCC-9 ${ }_{\text {CET-R }}$ ), we observed the accumulation of a subclone carrying a $M E T$ mutation which has been shown to be involved in cetuximab resistance [32]. The exact mechanisms of how these genetic alterations are involved in CCI-779 sensitivity have to be elucidated in future studies.

We next assessed if combinatorial treatment with CCI-779 and cisplatin or cetuximab increased growth inhibition in these cell line models of acquired drug resistance. Cisplatin-resistant cells with increased sensitivity to CCI-779 could be only slightly sensitized further by addition of cisplatin to CCI-779 only in the $\mathrm{FaDu}$ but not in the UD-SCC-4 model (Figure 7). Cetuximabresistant cells with reduced sensitivity to CCI-779 could be sensitized to CCI-779 by its combination with 


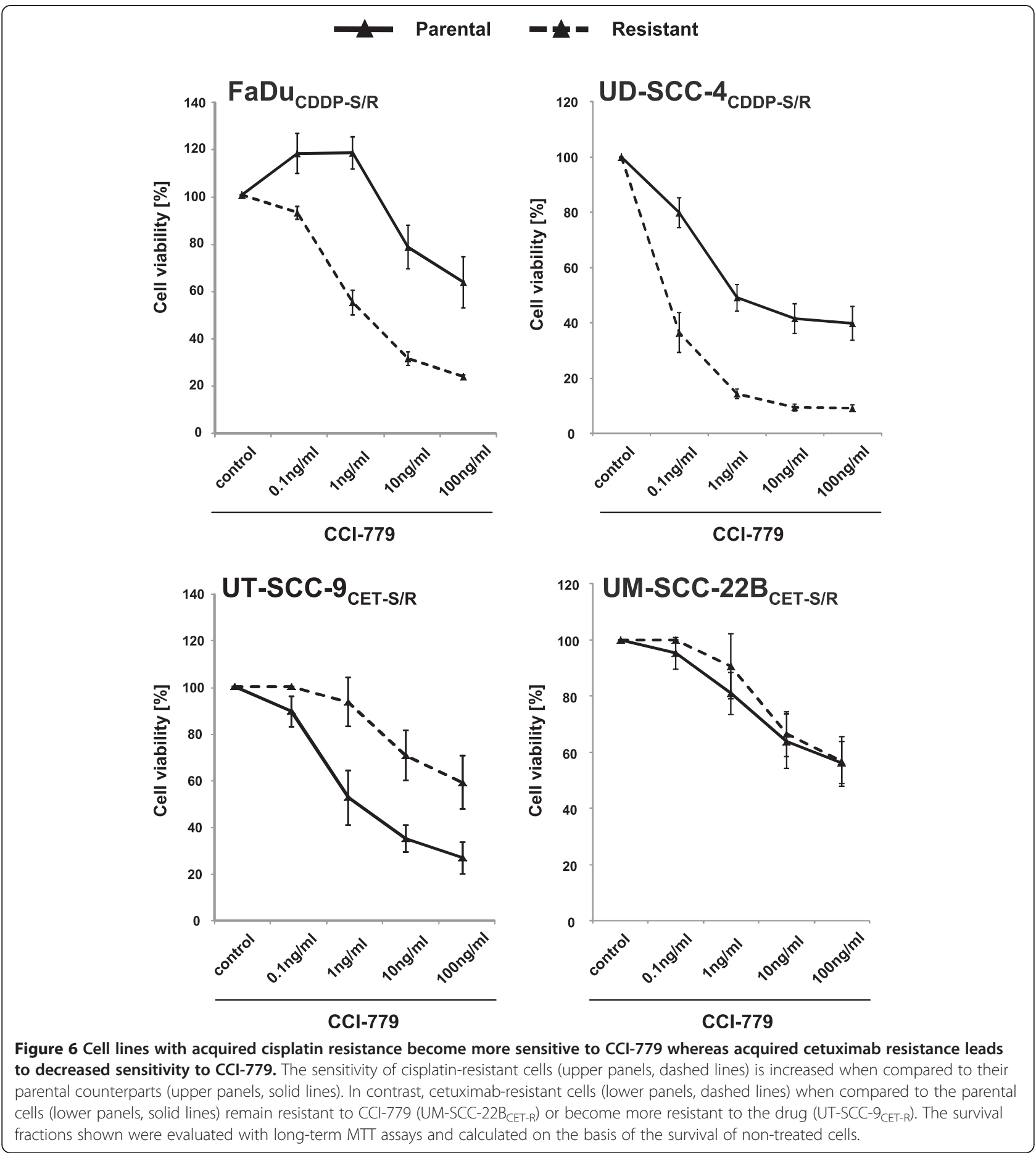

cetuximab in both cell line models of acquired cetuximab resistance (Figure 7).

These data suggest that patients with recurrent cisplatin-resistant tumors might benefit from mTORi treatment. In line with our results, a clinical trial in recurrent/metastatic HNSCC addressing the efficacy of temsirolimus after cisplatin treatment failure has shown promising results [33]. Further drug partners in this setting will have to be identified given the absence of any potentiating effects of the CCI-779/cisplatin combination. Treatment with the combination of cetuximab and mTORi, but not mTORi alone could be beneficial for patients with tumors with acquired cetuximabresistance. Such dual inhibition of EGFR and the 


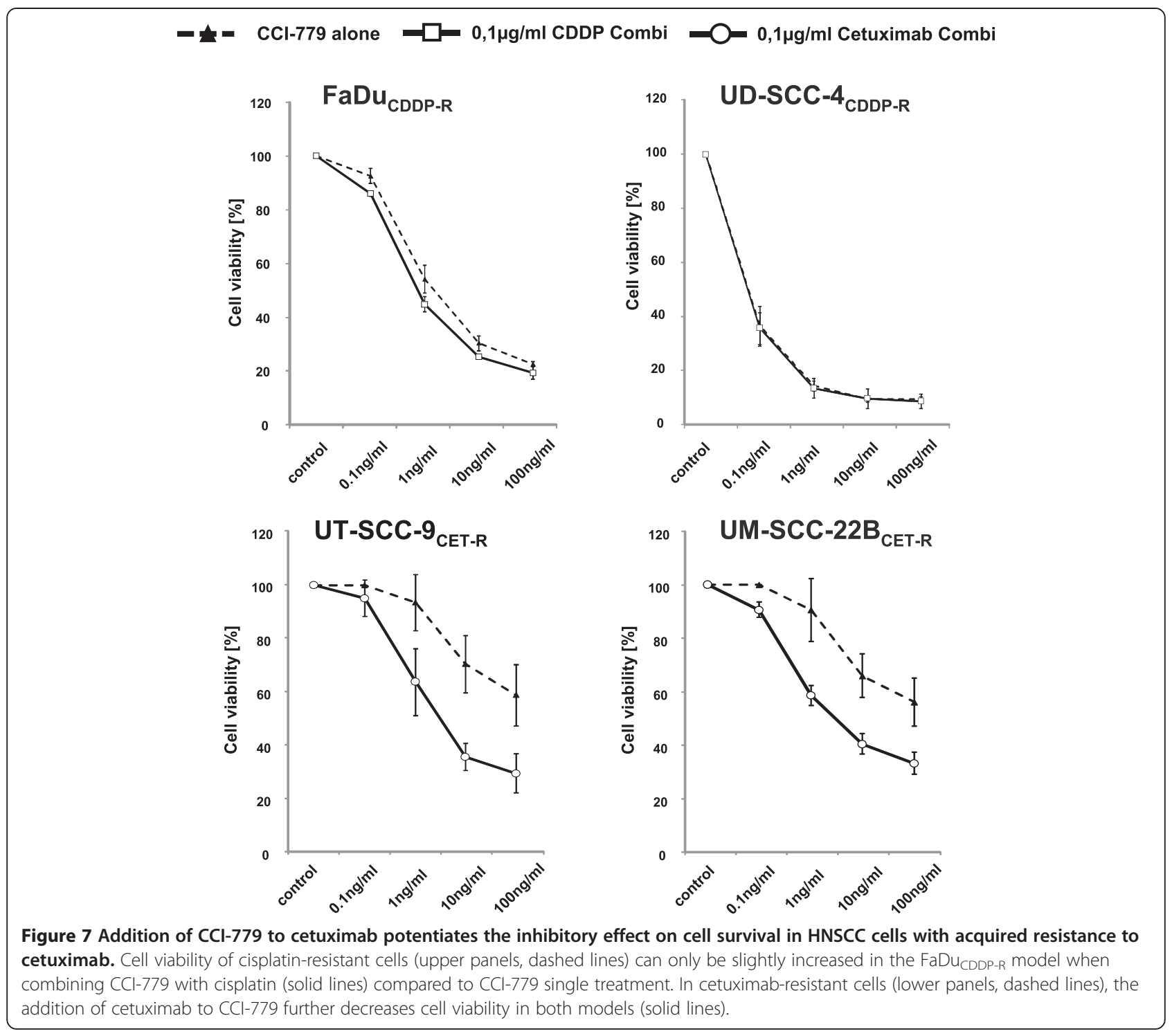

mTOR pathway is currently being tested and our study supports the need for further prospective trials in HNSCC.

\section{Conclusions}

The results from our study demonstrating no crossresistance of HNSCC cells to CCI-779 and cisplatin suggests that mTORi could serve as a potential treatment option in tumors with primary resistance to cisplatin. We identified EGFR/p-EGFR expression as potential negative predictive biomarker for mTORi efficacy and the combination of CCI-779 and cetuximab as a therapeutic regimen with increased growth-inhibitory potential. Our results from the preclinical models of acquired resistance also suggest clinical potential of mTORi in the recurrent, cisplatin-refractory setting and its combination with cetuximab in tumors with acquired resistance to EGFR blockade.

\section{Methods}

\section{Cell lines and reagents}

The HNSCC cell lines UD (University of Düsseldorf)SCC-4, -5, UT (University of Turku)-9, -15, -23, UM (University of Michigan)-SCC-11B, -17B, -22B, -25, -74B, and SCC-9 were a gift from T.K. Hoffmann (University of Essen) and T.E. Carey (University of Michigan) [34]. FaDu was purchased from ATCC. The identity of the cell lines was confirmed by high-throughput SNP-based authentication (Multiplexion, Heidelberg, Germany). All cell lines were tested for mycoplasma at monthly intervals by RT-PCR [35]. Contaminated cultures were treated with Mycoplasma Removal Agent (MP Biomedicals, Santa Ana, USA) according to the manufacturer's protocol. Cells were cultured in Minimal Essential Medium (MEM) supplemented with $10 \%$ heat-inactivated fetal bovine serum and $1 \times$ non-essential amino acids. Acquired 
resistance to cisplatin or cetuximab was attained by culturing the cells for a period of 4-9 months in increasing concentrations of the respective drug. All cell culture reagents were from GIBCO (life technologies, Carlsbad, CA, US). Cell cultures were incubated at $37^{\circ} \mathrm{C}$ and $5 \% \mathrm{CO} 2$ in a humidified atmosphere. CCI-779 was provided by Pfizer (Berlin, Germany) and diluted in dimethylsulfoxide (DMSO) to a stock of $1 \mathrm{mg} / \mathrm{ml}$. Cisplatin was purchased from Sigma-Aldrich (Munich, Germany) and cetuximab was provided by Merck (Darmstadt, Germany). Working solutions were freshly prepared from the stock solution by dilution in cell culture medium on the day of the experiment.

\section{MTT viability assays}

Cells were seeded into 96-well plates at a density of 250300 cells/well. Twenty-four hours after seeding, cells were treated with CCI-779, cisplatin, cetuximab, or the combination. Cells were then incubated for $72 \mathrm{~h}$ (short-term) or 7 days (long-term). At the end of the experiments 3-(4,5dimethylthiazol-2-yl)-2,5-diphenyltetrazolium bromide reagent (MTT) was added to the cells and after one hour incubation formazan complexes were dissolved in DMSO and absorbance measured with a spectrophotometer. Survival fractions for given treatments were calculated on the basis of survival of untreated cells. Each sample was done in sextuplets and at least three independent experiments were carried out. From the dose-effect curves the IC50 values for cisplatin were calculated.

\section{Immunoblotting analysis}

Expression levels of phosphorylated and total proteins in cell lysates of HNSCC cell lines, either untreated or treated with $100 \mathrm{ng} / \mathrm{ml} \mathrm{CCI-779}$ were assessed by standard Western blot analysis. Briefly, cells were harvested by scraping in RIPA buffer. Standard SDS-polyacrylamide gel electrophoresis was performed using $40 \mu \mathrm{g}$ of total protein per sample, followed by transfer to PVDF membranes (Millipore, Billerica, MA, US). For detection, the following antibodies were used: p-EGFR Tyr1068 (\#2234), p-Erk Thr202/Tyr204 (clone D13.14.4E), Erk (clone 137 F5), p-S6 Ser235/236 (clone D57.2.2E), S6 (clone 5G10), p-S6K Thr389 (clone 108D2), S6K (clone 49D7) and GAPDH (clone 14C10), all from Cell Signaling Technology (Danvers, MA, US). The antibody against p53 (clone DO-1) was purchased from Santa Cruz (Santa Cruz, CA, US) and anti-EGFR (clone 13) from BD Biosciences (Franklin Lakes, NJ, US). Secondary antibodies included peroxidaseconjugated goat anti-mouse and goat anti-rabbit both from Jackson ImmunoResearch Laboratories (West Grove, PA, US). The immunoreactivity was detected using the Pierce ECL Plus Western Blotting Substrate (Thermo Scientific, Waltham, MA, US).

\section{Flow cytometry}

Cells were harvested by trypsinization and fixed in fixation buffer (Nordic MUbio, Susteren, Netherlands). Cells were incubated with primary and secondary antibodies in permeabilization buffer (Nordic MUbio) for $20 \mathrm{~min}$ each and were subsequently analyzed using the FACSCanto II cytometer and the FACSDiva Software v6 (BD Biosciences). The following antibodies were used for staining: rabbit anti-p-EGFR Tyr1068 (cell signaling), rabbit IgG isotype control and goat anti-rabbit FITC (Life Technologies, Darmstadt, Germany). Median specific staining intensity (MFI) was calculated as ratio of the fluorescence intensity of cells stained with the specific antibody and the isotype control.

\section{Sequencing analysis}

RNA was isolated and TP53 transcript expression analysis as well as Sanger sequencing was performed (Source BioScience, Berlin, Germany). For the panel-based next generation sequencing experiments, library preparation and semiconductor sequencing was performed as follows. Using the multiplex PCR based Ion Torrent approach as described previously [36,37], for this study an in-house gene panel for 45 HNSCC-related cancer genes was designed (see Additional file 1) using the COSMIC database [38] as well as two publications on exome sequencing data of HNSCC tumor material $[39,40]$. DNA was extracted from all of the cell lines with the Invisorb Spin Tissue Mini Kit (Stratec, Birkenfeld, Germany). Amplicon library preparation was performed using approximately $10 \mathrm{ng}$ of DNA as advised by the manufacturer. Briefly, the DNA was mixed with the primer pool, containing all primers for generating the 224 amplicons and the AmpliSeq HiFi Master Mix, and transferred to a PCR cycler (BioRad, Munich, Germany). PCR cycling conditions were as follows: Initial denaturation: $99^{\circ} \mathrm{C}$ for $2 \mathrm{~min}$, cycling: 21 cycles at $99^{\circ} \mathrm{C}$ for $15 \mathrm{sec}$ and $60^{\circ} \mathrm{C}$ for $4 \mathrm{~min}$. Subsequent to the PCR reaction, primer end sequences were partially digested using the FuPa reagent as instructed, followed by the ligation of barcoded sequencing adapters (Ion Xpress Barcode Adapters, Life Technologies). The final library was purified using AMPure XP magnetic beads (Beckman Coulter, Krefeld, Germany) and quantified using qPCR (Ion Library Quantitation Kit, Life Technologies) on a StepOnePlus Instrument (Life Technologies). The individual libraries were diluted to a final concentration of $100 \mathrm{pM}$ and eight to ten libraries were pooled and processed to library amplification on Ion Spheres using the Ion OneTouch 2 instrumentation with the 200 bp chemistry. Unenriched libraries were quality-controlled using Ion Sphere quality control measurement on a QuBit instrument. After library enrichment (Ion OneTouch ES), the library was processed for sequencing using the Ion Torrent $200 \mathrm{bp}$ 
sequencing chemistry and the barcoded eight to ten libraries were loaded onto a single 318 chip.

Raw data analysis was performed using Ion Torrent Software Suite (Version 3.6 and 4.0). The reads were aligned to the human reference sequence build 38 (hg19) using the TMAP aligner implemented in the Torrent Suite software. Detection of single base pair variants and insertion-deletion polymorphisms (InDels) compared to the human reference sequence was performed using either Ion Torrent Variant Caller (3.6 and 4.0). Detection thresholds for SNPs and InDels were set at an allele frequency of 5\%. Variants were annotated and filtered against the dbSNP and COSMIC databases and screened for possible splice site effects using the CLC genomics Suite 6 (CLCbio, Aarhus, Denmark). Copy number variations were determined using the coverage analysis plug-in of the Torrent Suite software.

\section{Transcript expression analysis}

Basal and irradiation-induced p21 expression levels, as read-out for p53 transcriptional activity in HNSCC cell lines, as well as basal EGFR levels were determined by quantitative reverse-transcriptase polymerase chain reaction (qRT-PCR). Total cellular RNA extraction was performed using the High Pure RNA Isolation Kit (Roche, Basel, Switzerland). Synthesis of cDNA was done with the Omniscript Reverse Transcription kit (QIAGEN, Hamburg, Germany), according to the supplied protocol, using random hexamers and oligo dT15 primers (Roche) and $2 \mu \mathrm{g}$ of total RNA. The quality of RNA was checked by GAPDH PCR and only samples positive for GAPDH transcripts were used for analysis. Real-time-PCR was performed in a reaction volume of $20 \mu \mathrm{l}$ containing $2 \mu \mathrm{l} \mathrm{cDNA}$, Light Cycler TaqMan Master (Roche), primers and probes for p21, EGFR and the housekeeping gene porphobilinogen deaminase $(P B G D)$ in concentrations recommended by the manufacturer (Real Time Ready Assays, Roche). PCR cycling was performed using a Light Cycler (Roche). Relative quantification of p21 and EGFR expression was done by normalization to the expression levels of PBGD.

\section{Statistical analysis}

All statistical analyses were performed using SPSS v.20.0 (IBM Corp., Armonk, NY, USA) software. The significance of differences in the cell survival fraction after treatment with $100 \mathrm{ng} / \mathrm{ml}$ temsirolimus of cell lines exhibiting diverse characteristics regarding TP53 was determined using the independent-samples $t$-test. The level of significance was set at $\mathrm{p}<0.05$.

\section{Additional file}

Additional file 1: HNSCC-Panel.

\section{Competing interests}

This project has been supported by PFIZER Pharma GmbH, by the German Cancer Consortium (DKTK) and a grant from the German Cancer Aid (\#108791, to I.T.).

\section{Authors' contributions}

IT supervised the project, helped designing the experiments and analysing the data. FN designed and performed experiments, analysed data and wrote the manuscript. WW and AS performed and analysed the DNA-sequencing experiments. VB contributed to the analysis of the data. All authors discussed the results and implications, commented on the manuscript at all stages and agreed on the content of the manuscript.

\section{Author details}

${ }^{1}$ Translational Radiooncology Laboratory, Department of Radiooncology and Radiotherapy, Charité University Hospital, Berlin, Germany. ${ }^{2}$ German Cancer Consortium (DKTK), German Cancer Research Center (DKFZ) Heidelberg, Partner Site Berlin, Berlin, Germany. ${ }^{3}$ Institute of Pathology, University Hospital Heidelberg, Heidelberg, Germany. ${ }^{4}$ German Cancer Consortium (DKTK), German Cancer Research Center (DKFZ) Heidelberg, Partner Site Heidelberg, Heidelberg, Germany. ${ }^{5}$ Department of Radiooncology and Radiotherapy, Translational Radiooncology Laboratory,

Charité-Universitätsmedizin Berlin, Charitéplatz 1, 10117 Berlin, Germany.

Received: 5 November 2014 Accepted: 10 March 2015

Published online: 01 April 2015

\section{References}

1. Parkin DM, Bray F, Ferlay J, Pisani P. Global cancer statistics, 2002. CA Cancer J Clin. 2005;55:74-108.

2. Kamangar F, Dores GM, Anderson WF. Patterns of cancer incidence, mortality, and prevalence across five continents: defining priorities to reduce cancer disparities in different geographic regions of the world. J Clin Oncol. 2006;24:2137-50.

3. Bonner JA, Harari PM, Giralt J, Cohen RB, Jones CU, Sur RK, et al. Radiotherapy plus cetuximab for locoregionally advanced head and neck cancer: 5-year survival data from a phase 3 randomised trial, and relation between cetuximab-induced rash and survival. Lancet Oncol. 2010;11:21-8.

4. Bossi $P$, Locati L, Licitra L. Emerging tyrosine kinase inhibitors for head and neck cancer. Expert Opin Emerg Drugs. 2013;18:445-59.

5. Gilbert J, Lee JW, Argiris A, Haigentz Jr M, Feldman LE, Jang M, et al. Phase II 2-arm trial of the proteasome inhibitor, PS-341 (bortezomib) in combination with irinotecan or PS-341 alone followed by the addition of irinotecan at time of progression in patients with locally recurrent or metastatic squamous cell carcinoma of the head and neck (E1304): a trial of the Eastern Cooperative Oncology Group. Head Neck. 2013;35:942-8.

6. Metwally MA, Frederiksen KD, Overgaard J. Compliance and toxicity of the hypoxic radiosensitizer nimorazole in the treatment of patients with head and neck squamous cell carcinoma (HNSCC). Acta Oncol. 2014;53:654-61.

7. Yao M, Galanopoulos N, Lavertu P, Fu P, Gibson M, Argiris A, et al. A phase II study of bevacizumab in combination with docetaxel and radiation in locally advanced squamous cell carcinoma of the head and neck. 2014. doi:10.1002/hed.23813. [Epub ahead of print].

8. Lui WW, Hedberg ML, Li H, Vangara BS, Pendleton K, Zeng Y, et al. Frequent mutation of the PI3K pathway in head and neck cancer defines predictive biomarkers. Cancer Discov. 2013;3:761-9.

9. Populo H, Lopes JM, Soares P. The mTOR signalling pathway in human cancer. Int J Mol Sci. 2012;13:1886-918.

10. Vilar E, Perez-Garcia J, Tabernero J. Pushing the envelope in the mTOR pathway: the second generation of inhibitors. Mol Cancer Ther. 2011;10:395-403.

11. Aissat N, Le Tourneau C, Ghoul A, Serova M, Bieche I, Lokiec F, et al. Antiproliferative effects of rapamycin as a single agent and in combination with carboplatin and paclitaxel in head and neck cancer cell lines. Cancer Chemother Pharmacol. 2008;62:305-13.

12. Sun ZJ, Zhang L, Hall B, Bian Y, Gutkind JS, Kulkarni AB. Chemopreventive and chemotherapeutic actions of mTOR inhibitor in genetically defined head and neck squamous cell carcinoma mouse model. Clin Cancer Res. 2012;18:5304-13.

13. Coppock JD, Wieking BG, Molinolo AA, Gutkind JS, Miskimins WK, Lee JH. Improved clearance during treatment of HPV-positive head and neck cancer through mTOR inhibition. Neoplasia. 2013;15:620-30. 
14. Molinolo AA, Marsh C, El Dinali M, Gangane N, Jennison K, Hewitt S, et al. mTOR as a molecular target in HPV-associated oral and cervical squamous carcinomas. Clin Cancer Res. 2012;18:2558-68.

15. Schedel F, Pries R, Thode B, Wollmann B, Wulff S, Jocham D, et al. mTOR inhibitors show promising in vitro activity in bladder cancer and head and neck squamous cell carcinoma. Oncol Rep. 2011;25:763-8.

16. Li Q, Song XM, Ji YY, Jiang H, Xu LG. The dual mTORC1 and mTORC2 inhibitor AZD8055 inhibits head and neck squamous cell carcinoma cell growth in vivo and in vitro. Biochem Biophys Res Commun. 2013;440:701-6.

17. Bauman JE, Arias-Pulido H, Lee SJ, Fekrazad MH, Ozawa H, Fertig E, et al. A phase II study of temsirolimus and erlotinib in patients with recurrent and/ or metastatic, platinum-refractory head and neck squamous cell carcinoma. Oral Oncol. 2013:49:461-7.

18. Cassell A, Freilino ML, Lee J, Barr S, Wang L, Panahandeh MC, et al. Targeting TORC1/2 enhances sensitivity to EGFR inhibitors in head and neck cancer preclinical models. Neoplasia. 2012;14:1005-14.

19. Ekshyyan $O$, Rong $Y$, Rong X, Pattani KM, Abreo F, Caldito G, et al. Comparison of radiosensitizing effects of the mammalian target of rapamycin inhibitor $\mathrm{CCl}-779$ to cisplatin in experimental models of head and neck squamous cell carcinoma. Mol Cancer Ther. 2009;8:2255-65.

20. Pickhard AC, Margraf J, Knopf A, Stark T, Piontek G, Beck C, et al. Inhibition of radiation induced migration of human head and neck squamous cell carcinoma cells by blocking of EGF receptor pathways. BMC Cancer. 2011;11:388

21. Fury MG, Lee NY, Sherman E, Ho AL, Rao S, Heguy A, et al. A phase 1 study of everolimus + weekly cisplatin + intensity modulated radiation therapy in head-and-neck cancer. Int J Radiat Oncol Biol Phys. 2013;87:479-86.

22. Herzog A, Bian Y, Vander Broek R, Hall B, Coupar J, Cheng H, et al. PI3K mTOR inhibitor PF-04691502 antitumor activity is enhanced with induction of wild-type TP53 in human xenograft and murine knockout models of head and neck cancer. Clin Cancer Res. 2013;19:3808-19.

23. Di Nicolantonio F, Arena S, Tabernero J, Grosso S, Molinari F, Macarulla T, et al. Deregulation of the PI3K and KRAS signaling pathways in human cancer cells determines their response to everolimus. J Clin Invest. 2010;120:2858-66.

24. Satheesha S, Cookson VJ, Coleman LJ, Ingram N, Madhok B, Hanby AM, et al. Response to mTOR inhibition: activity of elF4E predicts sensitivity in cell lines and acquired changes in elF4E regulation in breast cancer. Mol Cancer. 2011;10:19.

25. D'Amato $V$, Rosa R, D'Amato C, Formisano L, Marciano R, Nappi L, et al. The dual PI3K/mTOR inhibitor PKI-587 enhances sensitivity to cetuximab in EGFR-resistant human head and neck cancer models. Br J Cancer. 2014;110:2887-95.

26. Jimeno A, Kulesza P, Wheelhouse J, Chan A, Zhang X, Kincaid E, et al. Dual EGFR and mTOR targeting in squamous cell carcinoma models, and development of early markers of efficacy. Br J Cancer. 2007;96:952-9.

27. Chawla A, Adkins D, Worden F, Rao K, Hu H, Rowe Price K, et al. Effect of the addition of temsirolimus to cetuximab in cetuximab-resistant head and neck cancers: Results of the randomized PII MAESTRO study. 2014 ASCO Annual Meeting General Poster Session. J Clin Oncol. 2014;32:5s. suppl; abstr 6089.

28. Gadhikar MA, Sciuto MR, Alves MV, Pickering CR, Osman AA, Neskey DM, et al. Chk1/2 inhibition overcomes the cisplatin resistance of head and neck cancer cells secondary to the loss of functional p53. Mol Cancer Ther. 2013;12:1860-73

29. Yang F, Tang X, Riquelme E, Behrens C, Nilsson MB, Giri U, et al. Increased VEGFR-2 gene copy is associated with chemoresistance and shorter survival in patients with non-small-cell lung carcinoma who receive adjuvant chemotherapy. Cancer Res. 2011;71:5512-21.

30. Lu T, Jackson MW, Wang B, Yang M, Chance MR, Miyagi $M$, et al. Regulation of NF-kappaB by NSD1/FBXL11-dependent reversible lysine methylation of p65. Proc Natl Acad Sci U S A. 2010;107:46-51.

31. Oiso S, Ikeda R, Nakamura K, Takeda Y, Akiyama S, Kariyazono H. Involvement of NF-kappaB activation in the cisplatin resistance of human epidermoid carcinoma KCP-4 cells. Oncol Rep. 2012;28:27-32.

32. Krumbach $R$, Schuler J, Hofmann M, Giesemann T, Fiebig HH, Beckers T. Primary resistance to cetuximab in a panel of patient-derived tumour xenograft models: activation of MET as one mechanism for drug resistance. Eur J Cancer. 2011:47:1231-43.

33. Grünwald VKU, Boehm A, Guntinas-Lichius O, Hennemann B, Schmoll HJ, Ivanyi $P$, et al. Temsirolimus is active in refractory squamous cell carcinoma of the head and neck (SCCHN) failing platinum-based chemotherapy and cetuximab: efficacy and toxicity data from the phase II TEMHEAD study. Vienna. Austria: Annals of Oncology Abstract Book of the 37th ESMO Congress; 2012. p. 336.

34. Hoffmann TK, Sonkoly E, Hauser U, van Lierop A, Whiteside TL, Klussmann $J P$, et al. Alterations in the p53 pathway and their association with radio- and chemosensitivity in head and neck squamous cell carcinoma. Oral Oncol. 2008:44:1100-9.

35. van Kuppeveld FJ, Johansson KE, Galama JM, Kissing J, Bolske G, van der Logt JT, et al. Detection of mycoplasma contamination in cell cultures by a mycoplasma group-specific PCR. Appl Environ Microbiol. 1994;60:149-52.

36. Stenzinger A, Endris V, Pfarr N, Andrulis M, Johrens K, Klauschen F, et al. Targeted ultra-deep sequencing reveals recurrent and mutually exclusive mutations of cancer genes in blastic plasmacytoid dendritic cell neoplasm. Oncotarget. 2014;5:6404-13.

37. Endris V, Penzel R, Warth A, Muckenhuber A, Schirmacher P, Weichert W. Molecular diagnostic profiling of lung cancer specimens with a semiconductor-based massive parallel sequencing approach: feasibility, costs, and performance compared with conventional sequencing. J Mol Diagn. 2013;15:765-75

38. Forbes SA, Bindal N, Bamford S, Cole C, Kok CY, Beare D, et al. COSMIC: mining complete cancer genomes in the Catalogue of Somatic Mutations in Cancer. Nucleic Acids Res. 2011;39:D945-50

39. Stransky N, Egloff AM, Tward AD, Kostic AD, Cibulskis $K$, Sivachenko A, et al The mutational landscape of head and neck squamous cell carcinoma. Science. 2011;333:1157-60.

40. Agrawal N, Frederick MJ, Pickering CR, Bettegowda C, Chang K, Li RJ, et al. Exome sequencing of head and neck squamous cell carcinoma reveals inactivating mutations in NOTCH1. Science. 2011;333:1154-7.

41. Ng PC, Henikoff S. SIFT: Predicting amino acid changes that affect protein function. Nucleic Acids Res. 2003:31:3812-4.

42. Ramensky V, Bork P, Sunyaev S. Human non-synonymous SNPs: server and survey. Nucleic Acids Res. 2002;30:3894-900.

\section{Submit your next manuscript to BioMed Central and take full advantage of:}

- Convenient online submission

- Thorough peer review

- No space constraints or color figure charges

- Immediate publication on acceptance

- Inclusion in PubMed, CAS, Scopus and Google Scholar

- Research which is freely available for redistribution 